\title{
Performance of a Carbon Nanotube Field Emission Electron Gun
}

\author{
Stephanie A. Getty*a, Todd T. King ${ }^{\mathrm{a}}$, Rachael A. Bis ${ }^{\mathrm{a}}$, Hollis Jones ${ }^{\mathrm{b}}$, Federico Herrero ${ }^{\mathrm{b}}$, Bernard A. \\ Lynch $^{\mathrm{b}}$, Patrick Roman ${ }^{\mathrm{b}}$, and Paul Mahaffy ${ }^{\mathrm{c}}$ \\ ${ }^{2}$ NASA Goddard Space Flight Center, Materials Engineering Branch, Code 541, Greenbelt, MD \\ 20771; \\ bNASA Goddard Space Flight Center, Detectors Systems Branch, Code 553, Greenbelt, MD 20771; \\ 'NASA Goddard Space Flight Center, Atmospheric Experiments Laboratory, Code 699, Greenbelt, \\ MD 20771
}

\begin{abstract}
A cold cathode field emission electron gun (e-gun) based on a patterned carbon nanotube (CNT) film has been fabricated for use in a miniaturized reflectron time-of-flight mass spectrometer (RTOF MS). Performance of the CNT e-gun has been evaluated. A traditional thermionic electron gun has also been fabricated and evaluated in parallel and its performance is used as a benchmark in the evaluation of our CNT e-gun. Implications for future improvements and integration into the RTOF MS are discussed.
\end{abstract}

Keywords: Carbon nanotube, field emission, mass spectrometry

\section{INTRODUCTION}

Cold cathode field emission may be an attractive method of generating an energetically clean, low contamination, low power electron beam for the electron impact ionization component of a time-of-flight mass spectrometer. Traditional thermionic electron emitters are low-cost and have extensive heritage in terrestrial and spaceflight applications. However, they typically consume several watts of power [1] and their high temperature of operation produces undesired background gases that may interfere with measurements in a planetary environment.

In contrast, cold cathode field emitters, such as Spindt emitters [2], operate at lower temperatures and dissipate far less power. To date, however, Spindt emitters, commonly fabricated of silicon, have found limited use in electron gun applications, owing to the tendency of the high field tip region to be mechanically sputtered by the ionized species [3]. The mechanical dulling of the emission tip leads to short lifetimes and rapid degeneration of the emission current.

Recently, the field emission properties of carbon nanotubes (CNTs) have been the subject of extensive experimental and theoretical work [4-16] for applications in field emission displays, microwave power tube amplifiers, and electron microscopy [17-20]. The $s p^{2}$ hybridized carbon network is appealing in its chemical and mechanical robustness [21], compared to silicon, and in the geometry reported herein, allows for high emitter redundancy and long operating lifetime.

We are working to exploit these appealing properties of carbon nanotubes for use in a miniaturized reflectron time-offlight mass spectrometer that uses MEMS and nanofabrication techniques to accomplish full functionality in a low mass, power, and footprint package. The electron impact ionization source is the focus of this work, and we report on fabrication of a carbon nanotube electron gun (CNT e-gun), with an integrated electron extraction grid and charged particle optics. Performance of the as-built electron gun has been simulated with a commercial software package, SIMION [22], allowing us to compare the operation of the CNT e-gun to an idealized case and predict future avenues for improvement.

The electron beam generated by our CNT e-gun was evaluated electronically using a triode configuration, as shown schematically in Figure 1, in which the emission current is extracted from the CNT cathode using a grid electrode in close proximity, modulated using a series of electrostatic lenses, and subsequently collected at an anode. In this work, lenses 1-7 are generally at $\mathrm{V}=0$; future work will use the individually addressable lenses to manipulate the beam shape and focus. Using a commercially available Beam Visualization System (BVS-1; Colutron Research Corporation), we have the capability to replace the anode with a charged particle imaging component to directly observe the shape and 
intensity of the electron beam. The shape can be recorded as a function of lens voltage configuration and compared to electrical characterization measurements and SIMION predictions.

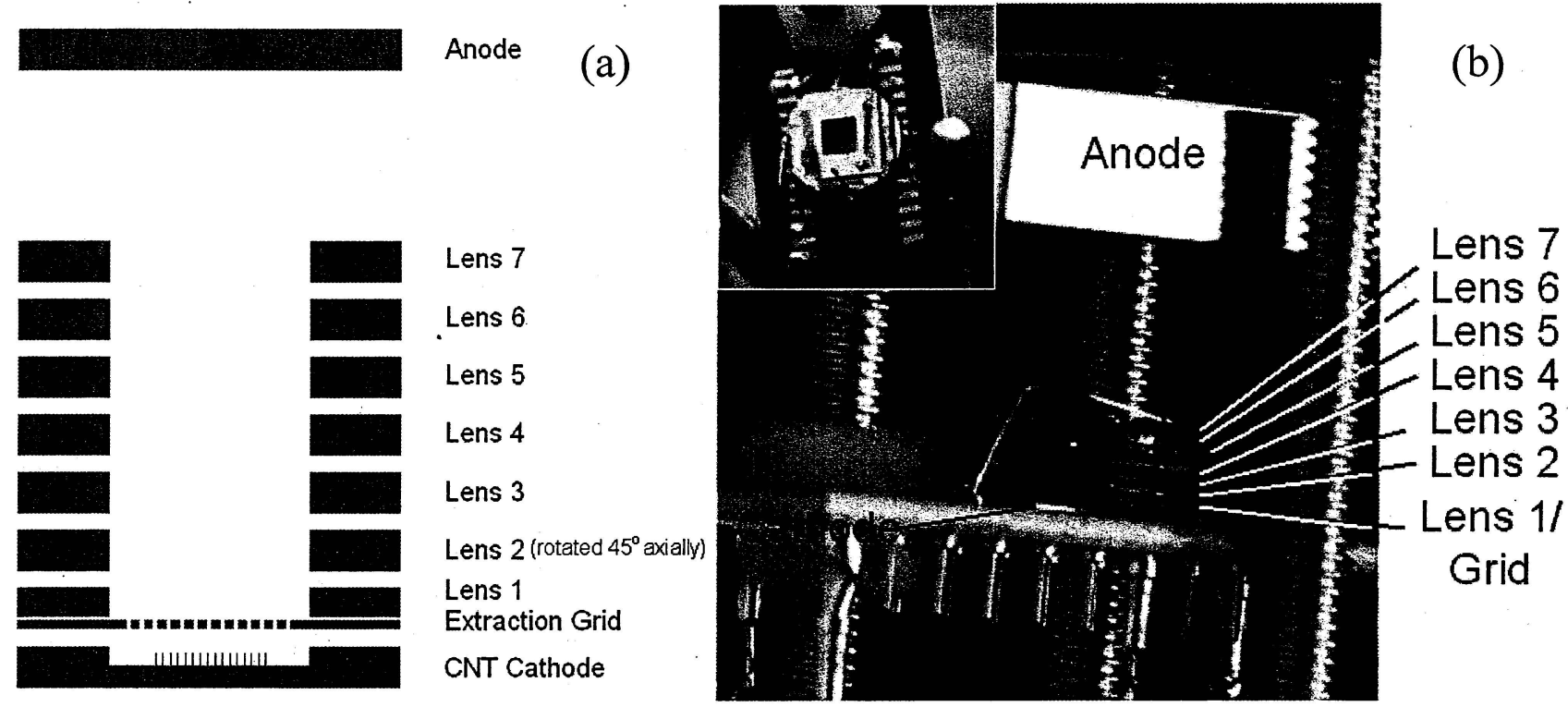

Figure 1. (a) Schematic of CNT e-gun, consisting of CNT cathode, extraction grid, electrostatic lenses 1-7, and anode. (b) Digital image of as-built CNT electron gun, side view, and top view (inset).

\section{METHODOLOGY}

\subsection{Simulation and Modeling}

An electrodynamics simulation software package, SIMION 3D Ion and Electron Optics Simulator was used to establish three-dimensional electrostatic potential array geometries, calculate the electric field of the free space between potential arrays, and simulate the flight of electrons within the model. The potential arrays define the geometry for all solid electrode structures in this design and were created by programming a geometry file. Note that insulating structures are not explicitly included in this model. Rather, insulating structures are treated as free space in the model for the following reasons: 1) most insulator materials selected for ion source designs have a dielectric constant close to that of vacuum $\left(\varepsilon_{0}=1\right)$, and thus have a small effect on the overall electric field, and 2) ion source designs typically position insulating surfaces away from direct impingement of charged particle beams to avoid surface charging effects.

The electric potential is related to the charge density in the system through the following relation:

$\Delta^{2} V=-\rho / \varepsilon_{0}=0$,

where $V$ is electric potential and $\rho$ is the charge density, which vanishes when space charge effects are neglected. SIMION adopts this simplification to ease computational requirements. The electric potential is then related to the electric field, $E$, by

$$
E=-\nabla V \text {. }
$$

The electric potential throughout the entire model was determined by solving the Laplace equation in the absence of space charge effects (Equation 1), via an over-relaxation finite difference technique at every non-electrode point subject to the boundary conditions imposed by the defined electrodes. The electric field was then computed by taking the gradient of the electric potential as shown in Equation 2. A fine grid spacing of $20 \mu \mathrm{m}$ per grid unit was defined for the entire model volume. 


\subsection{Fabrication and Assembly: Thermionic E-gun}

As a performance benchmark, we studied the performance of a traditional thermionic electron gun in parallel with the CNT e-gun development. The e-gun consisted of a half-cylindrical electrostatic repeller forming the base of the stack to aid focusing of the electron beam, a thoriated tungsten filament, two coincident rectangular metal apertures measuring $100 \mu \mathrm{m} \times 500 \mu \mathrm{m}$ separated by $1.6 \mathrm{~mm}$ of Macor insulator, a conductive electron gun exit lens, and a stainless steel anode or, alternatively, the BVS. A SIMION schematic of this assembly is shown in Figure 2 (a), and the as-built thermionic e-gun in Figure 2 (b).

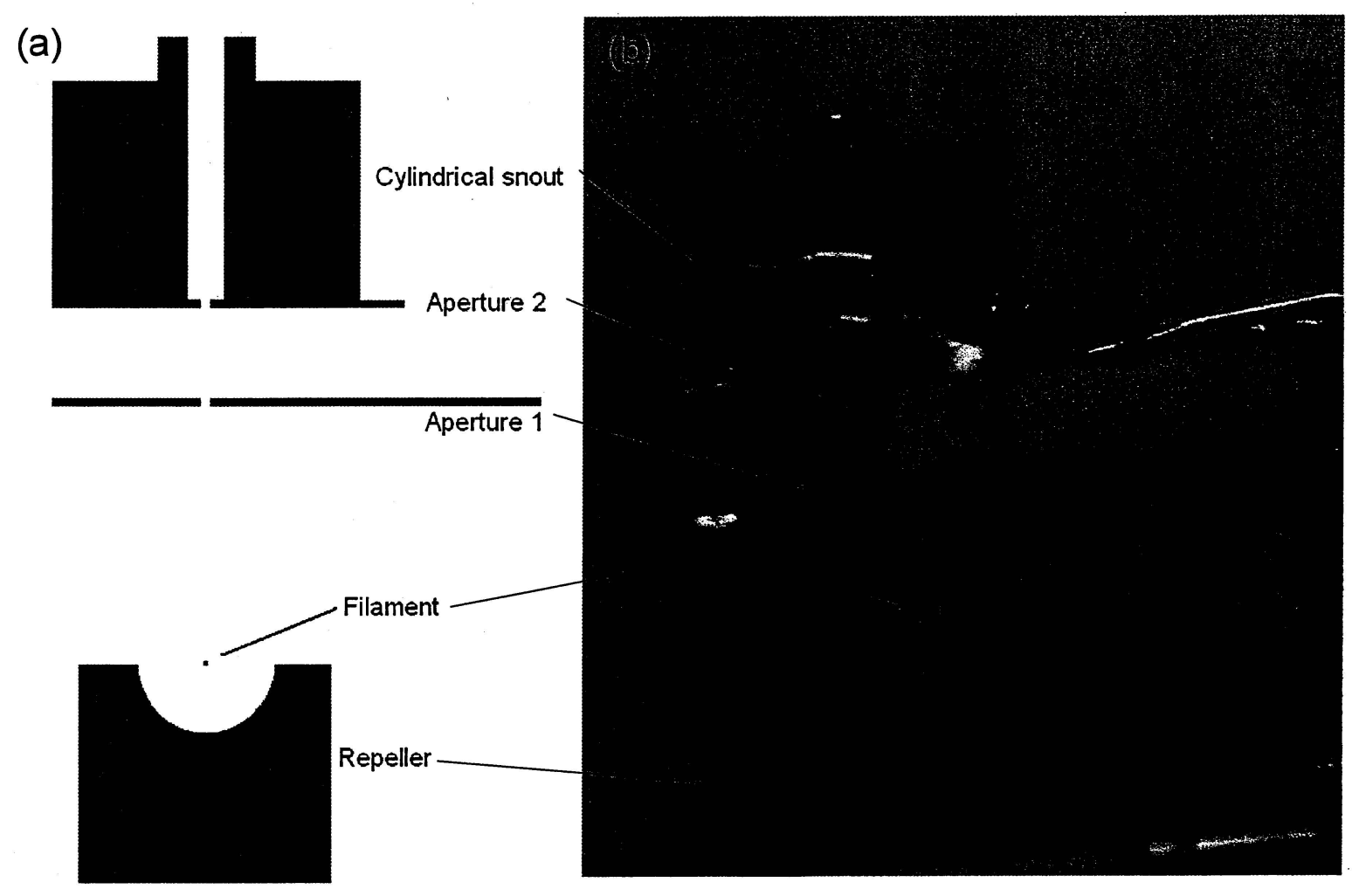

Figure 2. (a) Axial cross-section of thermionic e-gun with lens assembly. (b) Digital image of as-built thermionic e-gun mounted to test fixture.

\subsection{Fabrication and Assembly: CNT E-gun}

The CNT e-gun has a CNT cathode, silicon grid, seven silicon electrostatic lenses, and a stainless steel anode. To fabricate the CNT cathode, an oxidized silicon substrate was etched to define a $90 \mu \mathrm{m}$-deep recessed well, exposing bare silicon at the base of the well. The CNTs were grown in place using catalyst-assisted chemical vapor deposition (CVD), where the catalyst is a bi-layer of $10 \mathrm{~nm} \mathrm{Al} / 10 \mathrm{~nm} \mathrm{Fe} \mathrm{[23].} \mathrm{CVD} \mathrm{growth} \mathrm{was} \mathrm{performed} \mathrm{at} 750^{\circ} \mathrm{C}$ in $1000 \mathrm{sccm}$ flowing ethylene.

The CNT cathode consisted of a patterned film of CNTs on an array, with $50 \mu \mathrm{m}$ pitch and $5 \mu \mathrm{m} \times 5 \mu \mathrm{m} \times 10 \mu \mathrm{m}$ high CNT towers, as shown in Figure 3. The patterned catalyst is defined by electron beam lithography and subsequent thermal evaporation of the $\mathrm{Al} / \mathrm{Fe}$ bilayer prior to $\mathrm{CNT}$ growth. Electrical contact to the cathode is established through the silicon substrate. 




Figure 3. Scanning electron micrograph of patterned CNT array. Each CNT tower measures $5 \mu \mathrm{m} \times 5 \mu \mathrm{m} \times 10 \mu \mathrm{m}$

(height). The pitch of the array is $50 \mu \mathrm{m}$, and the entire array is $2 \mathrm{~mm} \times 2 \mathrm{~mm}$.

The grid component is fabricated in a $10 \mu \mathrm{m}$-thick device layer of a silicon on insulator (SOI) wafer using standard photolithography and silicon micromachining. Typical dimensions of the grid are $3 \mathrm{~mm} \times 3 \mathrm{~mm}$ grid area, with $5 \mu \mathrm{m}$ bar width and $50 \mu \mathrm{m}$ hole width. Electrostatic lenses 1 through 7 are SOI substrates, with a square through-hole of 3 $\mathrm{mm} \times 3 \mathrm{~mm}$ in the center for beam transmission. Thin film Au was thermally evaporated on the interior surfaces of the SOI lenses to prevent the electron beam from inducing charge build-up in the electrically insulating layer.

Figure 4 (a) shows a top view of the assembled CNT e-gun stack, and a high resolution optical micrograph in Figure 4 (b) reveals the array of CNT towers. The defocused grid component can be seen overlaying the CNT array.



Figure 4. (a) Optical stereomicroscope image of the CNT cathode and electrostatic lens assembly, top view. (b) High resolution electron micrograph of the patterned CNT array, visible as dark points through the defocused grid. 


\subsection{Electrical Study of Performance}

For electrical studies of both the CNT e-gun and the thermionic e-gun, performance was evaluated by collecting current at various points in the e-gun stack and ultimately at an anode. All measurements were performed in vacuum of order $10^{-6}$ torr or better. Emission and beam control were accomplished by applying voltages to each e-gun element, as directed by SIMION calculations. Quantitative measurements of anode current as a function of these voltages were compared to intensity measurements on the BVS to correlate e-gun performance.

For the thermionic e-gun, typical voltages applied to the repeller, cathode, aperture 1, aperture 2, electron gun exit lens, and anode are given in Table 1. Voltage was applied to the repeller using a SRS PS325 power supply. Voltage was applied to the cathode using a HP 6205B power supply, and collected current was sent through an external resistor (typically $100 \mathrm{k} \Omega$ or $1 \mathrm{M} \Omega$ ), and the voltage drop across the resistor was recorded.

For the CNT e-gun; typical cathode, grid, lens, and anode voltages are shown in Table 2. Cathode voltage was applied using an EMCO high voltage power supply or a Fluke 415B high voltage power supply. Lens voltages were applied using a HP 6205B power supply. Collected current was measured using the dropping resistor method, as in the case of the thermionic e-gun.

Table 1. Experimental voltage configuration of thermionic e-gun, as directed by SIMION simulations. The shape of the beam is expected to be rectangular.

\begin{tabular}{|cc|}
\hline Beam Shape & Rectangular \\
\hline Repeller Voltage & $-84 \mathrm{~V}$ \\
\hline Cathode Voltage & $-75 \mathrm{~V}$ \\
\hline Aperture 1 & $0 \mathrm{~V}$ \\
\hline Aperture 2 & $0 \mathrm{~V}$ \\
\hline E-gun Exit Lens & $0 \mathrm{~V}$ \\
\hline Anode Voltage & $0 \mathrm{~V}$ \\
\hline
\end{tabular}

Table 2. Experimental voltage configuration for CNT e-gun, as directed by SIMION simulations and experimental requirements. The shape of the beam is expected to be square.

\begin{tabular}{|cc|}
\hline Beam Shape & Square \\
\hline Cathode Voltage & $-700 \mathrm{~V}$ \\
\hline Grid Voltage & $0 \mathrm{~V}$ \\
\hline Lens 1 Voltage & $0 \mathrm{~V}$ \\
\hline Lens 2 Voltage & $0 \mathrm{~V}$ \\
\hline Lens 3 Voltage & $0 \mathrm{~V}$ \\
\hline Lens 4 Voltage & $0 \mathrm{~V}$ \\
\hline Lens 5 Voltage & $0 \mathrm{~V}$ \\
\hline Lens 6 Voltage & $0 \mathrm{~V}$ \\
\hline Lens 7 Voltage & $0 \mathrm{~V}$ \\
\hline Anode Voltage & $0 \mathrm{~V}$ \\
\hline
\end{tabular}




\subsection{Beam Visualization}

A commercially available beam visualization system (BVS) was used to amplify and project the generated electron beams onto a conflat-mounted viewport. The BVS consists of a chevron stack of two microchannel plate (MCP) detectors to first amplify the current and a phosphorescent screen (PS) to convert incident electrons into visible photons. Images were captured using a digital camera and integrated intensity levels were obtained using the Image Pro Plus image analysis software.

The rectangular geometry of the thermionic e-gun produced a rectangular beam. The imaging results, discussed further in the Results section below, were acquired using $1000 \mathrm{~V}$ across the MCP stack and $3500 \mathrm{~V}$ on the PS.

\section{DATA}

\subsection{Electrical Characterization: Thermionic E-gun}

We first discuss the thermionic e-gun electrical and beam imaging results to introduce the experimental techniques and results in a well understood conventional system. In this discussion, we refer to filament bias and current to be the voltage and current, respectively, across the filament, whereas cathode voltage denotes an additive voltage level of the filament relative to the other e-gun elements. The thoriated tungsten filament was typically operated at power dissipation levels of 100 to $300 \mathrm{~mW}$, with maximum anode current of $4 \times 10^{-8} \mathrm{~A}$. This value is used as a benchmark in the CNT e-gun discussion, below.

To investigate the effect of tuning the system voltages on the collected current, we measured the current first at aperture 1 (to approximate the total emitted current) and then the current transmitted through the aperture assembly at the anode as a function of repeller voltage and cathode voltage. The aperture and anode voltages were maintained at $0 \mathrm{~V}$ during these measurements.

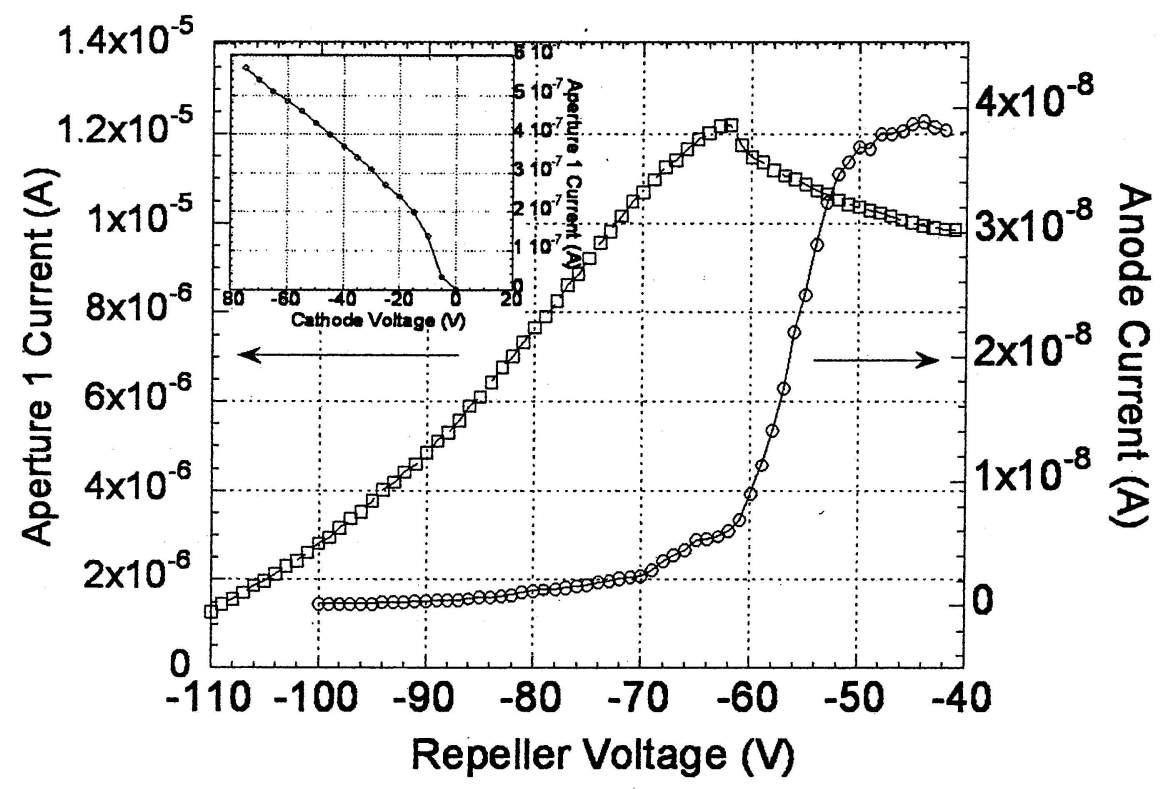

Figure 5. Emission current (A) collected at aperture 1 (squares; left axis) and at the anode (circles; right axis) as a function of repeller voltage $(\mathrm{V})$. Each curve shows a maximum, though occurring at different repeller voltages and having a different functional form. This mismatch is likely due to misalignment of the aperture assembly with the filament axis.

Current collected at aperture 1 and at the anode are shown in Figure 5 as a function of repeller voltage. The observed peak is predicted by SIMION calculations and is discussed further in the Results section. The inset shows the familiar diode-like behavior of aperture 1 current and anode current as a function of cathode voltage. 


\subsection{BVS Demonstration: Thermionic E-gun}

An image of the rectangular thermionic beam was acquired and is shown in Figure 6 (a). The beam is not well centered on the screen, due to a physical misalignment of the thermionic e-gun with respect to the screen. The direction of beam offset on the screen is consistent with the known offset of the e-gun. Nevertheless, a rectangular electron beam is clearly seen as a high-intensity glow on the PS. Some distortion is seen in the extreme lower left portion of the beam, likely due to electrostatic repulsion from a metal cylindrical guide, with radius of the PS, positioned between the e-gun assembly and the MCP/PS stack. A processed image is shown in Figure $6(\mathrm{~b})$ to highlight the beam shape.

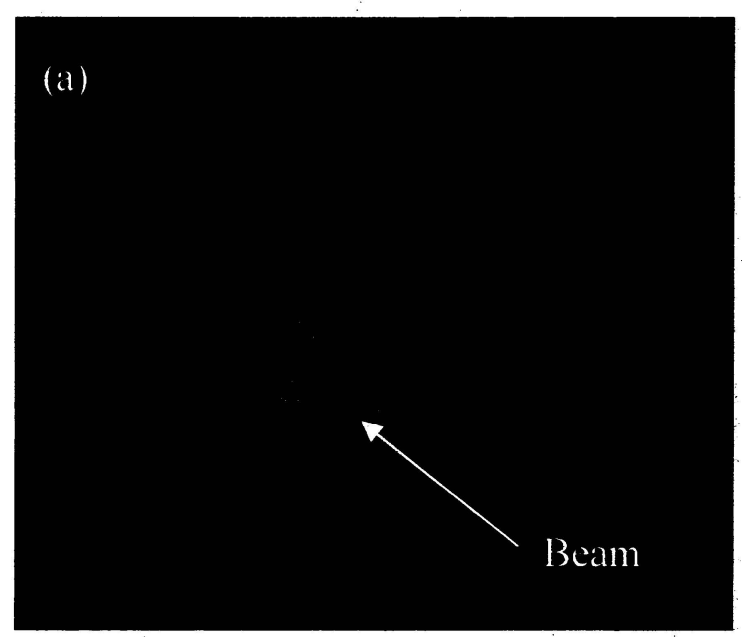

(b)

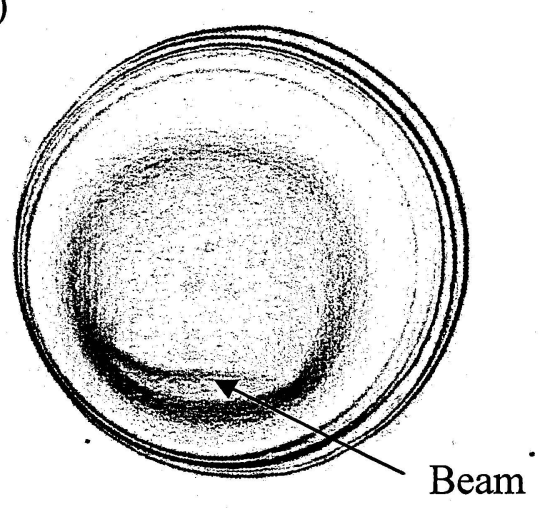

Figure 6. Digital image of the phosphorescent screen of the BVS during thermionic e-gun operation. An elongated region of high intensity indicates the location of the thermionic electron beam at the bottom of the screen (raw data). Processed image (Adobe Photoshop) reveals an enhanced view of the imaged beam.

The current collected within the imaged beam at the BVS is expected to follow the anode current, or the current transmitted through the aperture assembly, as measured in the previous section. However, delineation of the imaged beam is prone to large error in the integrated intensity, and integration was instead performed across the entire PS.

We observe that the entire PS glows, and we suggest that the BVS data include not only the beam transmitted through the aperture assembly but also spurious currents that escape to the BVS around the aperture assembly. This is likely due to the length of the filament, which is comparable to the dimensions of the aperture assembly. We therefore conclude that the PS intensity dependence on repeller and cathode voltages most closely follows the behavior of the total beam, i.e. the behavior followed by the emission current collected at aperture 1 .

\subsection{Electrical Characterization: CNT E-gun}

For a precursor device consisting of a patterned CNT array (as shown in Figure 2), an extraction grid, and an anode, emission current density is shown as a function of applied electric field in Figure 8. Current density is calculated using the total coverage of CNT towers, neglecting the open spaces between array elements. Electric field was estimated using the simple approximation of the voltage divided by the distance between the emitters and the grid, which was $275 \mu \mathrm{m}$ for the data shown in Figure 8.

Cold cathode field emission is well described by Fowler-Nordheim tunneling [24], given by the following relation:

$J=K_{1} E^{2} \exp \left(-\frac{K_{2}}{E}\right)$,

where $J$ is the current density, $\mathrm{K}_{1}$ and $\mathrm{K}_{2}$ are constants, and $E$ is the electric field. The inset of Figure 8 shows $\ln \left(\mathrm{J} / \mathrm{E}^{2}\right)$ versus $1 / \mathrm{E}$, and our data show good agreement with Fowler-Nordheim behavior. 

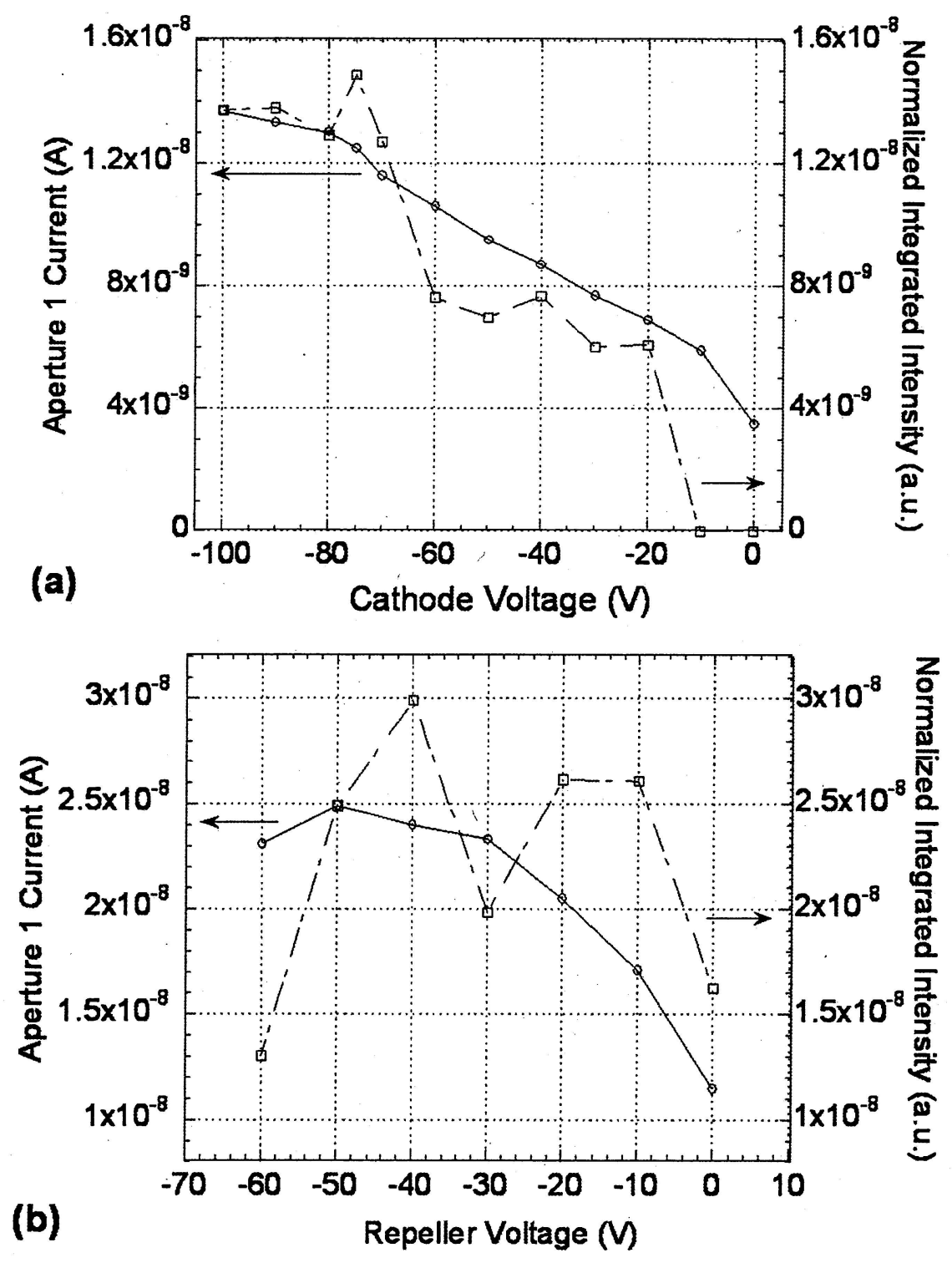

Figure 7. (a) Current at aperture 1 (A; circles; left axis) and normalized integrated intensity at PS (arbitrary units; squares; right axis) versus cathode voltage (V). (b) Current at aperture 1 (A; circles; left axis) and normalized integrated intensity at PS (arbitrary units; squares; right axis) versus repeller voltage (V). Although the error is large in our PS intensity data, qualitative agreement is seen for both measurements. 


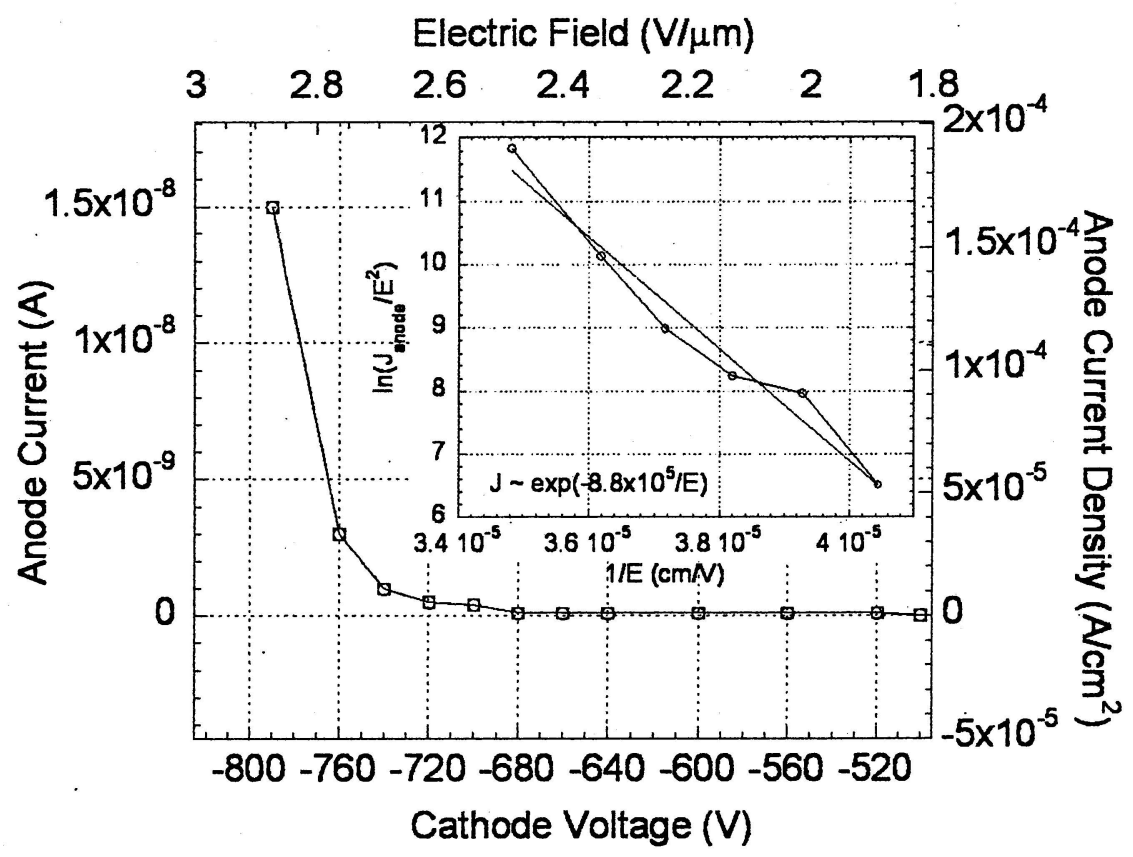

Figure 8. Emission current (A; left axis) shown versus extraction voltage (V; bottom axis), where the voltage is applied to the extraction grid, relative to the CNT cathode; also shown is emission current density $\left(\mathrm{A} / \mu \mathrm{m}^{2}\right.$; right axis) versus electric field ( $\mathrm{V} / \mu \mathrm{m}$; top axis). A fit to Fowler-Nordheim tunneling is shown in the inset.

Field emission turn-on, defined as the electric field required to generate $10 \mu \mathrm{A} / \mathrm{cm}^{2}$ of emission current density, was achieved at an applied electric field less than $2.5 \mathrm{~V} / \mu \mathrm{m}$, a value that is competitive with or better than other CNT field emission sources previously reported $[7,9,11]$. Threshold field is typically used to describe the electric field at which the emission current exceeds $10 \mathrm{~mA} / \mathrm{cm}^{2}$ [25]. This convention is related to the required current density to excite fluorescence from a phosphorescent screen in the field emission display industry. From the parameters obtained in our Fowler-Nordheim fit, we extrapolate the threshold field to be approximately $3.2 \mathrm{~V} / \mu \mathrm{m}$.

Table 3. Comparison of maximum measured anode current and corresponding power consumption levels for the thermionic e-gun and CNT e-gun.

\begin{tabular}{|ccc|}
\hline & Thermionic & CNT \\
\hline $\begin{array}{c}\text { Max. Current at } \\
\text { Anode }\end{array}$ & $4 \times 10^{-8} \mathrm{~A}$ & $1.5 \times 10^{-8} \mathrm{~A}$ \\
\hline $\begin{array}{c}\text { Power } \\
\text { Consumption }\end{array}$ & $100 \mathrm{~mW}$ & $0.8 \mathrm{~mW}$ \\
\hline
\end{tabular}

Here, we turn to an analysis of power consumption of the CNT e-gun, compared to that of the thermionic e-gun. For the $\mathrm{CNT}$ e-gun, maximum current detected at the grid was $1 \times 10^{-6} \mathrm{~A}$ (not shown) and at the anode was $1.5 \times 10^{-8} \mathrm{~A}$, for a maximum extraction voltage of $800 \mathrm{~V}$. This corresponds to a power dissipation of $0.8 \mathrm{~mW}$. A comparison to the power dissipation for the thermionic e-gun is given in Table 3. For these two prototype emitters, the CNT e-gun produces comparable levels of current, with a factor of 100 reduction in power dissipation. Improvements to both systems are expected in a future prototype assembly.

We note that spaceflight applications generally require tens to hundreds of microamps of emission current. It should be emphasized that the device presented here is an unoptimized device, with large separation between the CNT cathode tips and the extraction grid, such that applied electric field is limited by high voltage breakdown of our experimental fixture and does not reflect physical limitations of the CNT system. In fact, the CNT device shown here was previously tested, prior to lens integration, with approximately half the cathode-grid spacing shown here. In the previous configuration, we 
measured $50 \mu \mathrm{A}$ of current with an extraction voltage of only $200 \mathrm{~V}$, well within standard spaceflight operating conditions. We expect to achieve this performance in a future prototype integrated electron gun with further optimization and maturation of our fabrication and assembly techniques.

\subsection{Future Work: BVS Study of CNT E-gun}

Whereas electrical characterization gives confirmation of Fowler-Nordheim tunneling behavior in the CNT e-gun, enhanced data can be obtained using the beam visualization system. In future work, we plan to use the BVS to investigate the effects of different voltage configurations on the shape of the electron beam and compare to SIMION predictions. These measurements will allow verification of lens efficiency in our CNT e-gun system.

(a)



(b)

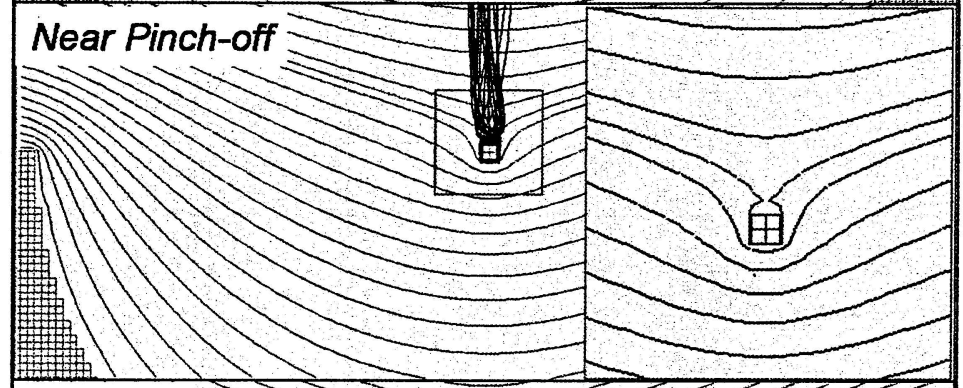

(c)



Figure 9. SIMION simulations of electron beam trajectories (blue) at various repeller voltages. Equipotential contours are shown in red. (a) Low repeller voltage produces a defocused beam. (b) As repeller voltage is increased, the equipotential contours curve around the filament. The green contour is near pinch-off; detail of this contour (without electron trajectories for clarity) is shown in the inset. (c) At large repeller voltages, the equipotentials pinch off to confine the electron beam in a potential well near the filament.

\section{RESULTS}

\subsection{Thermionic E-gun}

In Figure 5, maxima occur in the current measured at both collection points but the maxima are not located at the same repeller voltage setting. This reflects the mechanism generating the current maximum as a function of repeller voltage. . At low repeller voltage, depicted in Figure 9 (a), the beam current produced by the filament is highly defocused and reflects the cylindrical symmetry of the filament. As the repeller voltage is increased, as in Figure 9 (b), the beam is focused and "forward biased," i.e. the electrons are forced in the direction of the aperture stack. With further increase of the repeller voltage, shown in Figure 9 (c), the beam becomes highly collimated, until the equipotential contours of the 
repeller field "pinch off" to form a potential well. It is this pinch-off voltage that gives rise to a peak in the current. This is predicted by SIMION and qualitatively verified by our experimental data.

The data for current collected at aperture 1 are reproduced in Figure 10 to compare to simulated current data using SIMION. The SIMION results are normalized, at the peak current value, to the experimental data. Here, we compare simulated data collected at the anode to experimental data collected at aperture 1 . We believe that the misalignment of our physical stack and space charge effects not modeled by SIMION are responsible for the shift in the position of the maximum. Improved fabrication techniques will be implemented in future electron gun prototypes to minimize these spurious effects.

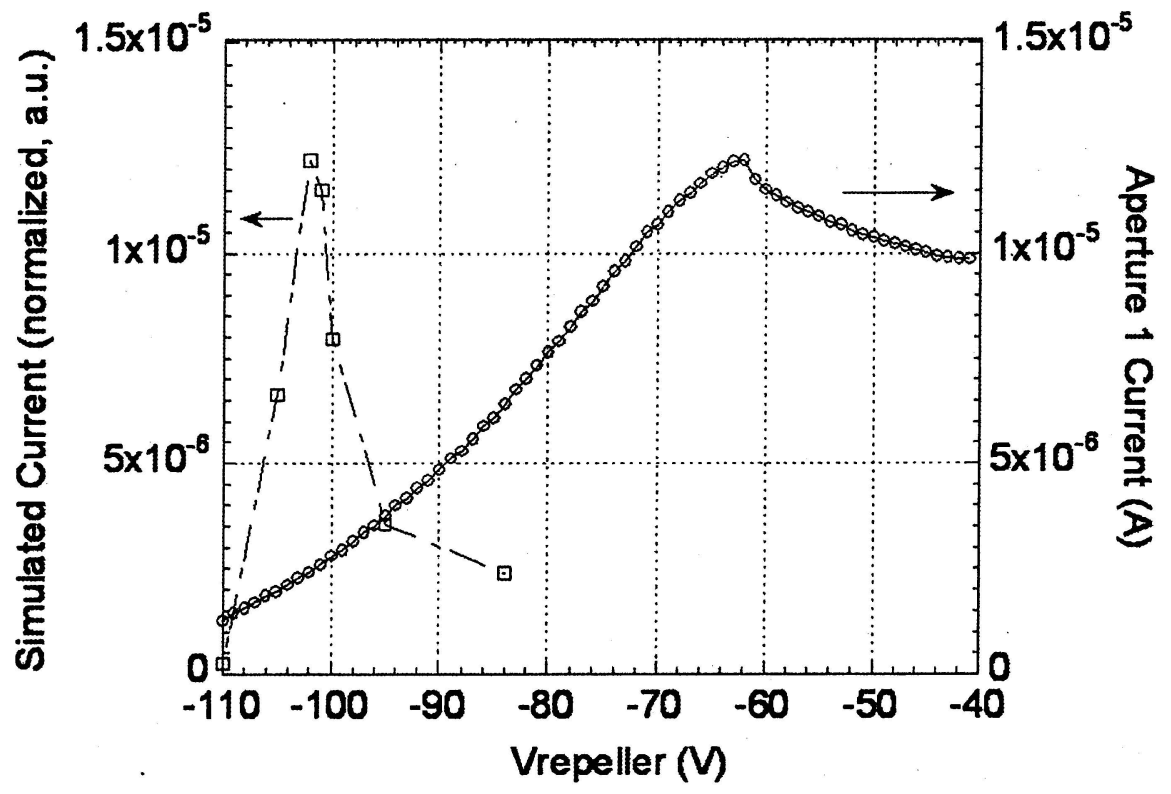

Figure 10. Aperture 1 current (A) versus repeller voltage (V) (circles, right axis). Also shown is simulated current using SIMION (squares; left axis), normalized to the maximum value of aperture 1 current, versus repeller voltage.

\subsection{CNT E-gun}

Our future work will include BVS measurements of the CNT e-gun field emission. The fit to Fowler-Nordheim behavior in Figure 8 allows an estimate of field enhancement factor. The prefactor of the slope is given by Equation 4 [9]:

$$
K_{2}=\frac{B \Phi^{3 / 2}}{\beta} \text {, }
$$

where $B$ is a constant given below, $\Phi$ is the work function, and $\beta$ is the field enhancement factor. Assuming an approximate work function of $5 \mathrm{eV}$ for graphite and a proportionality factor of $6.83 \times 10^{7} \mathrm{~V} \mathrm{eV}^{-3 / 2} \mathrm{~cm}^{-1}$, we obtain a field enhancement factor, $\beta$, of 900 . Although it has been shown that the work function of carbon nanotubes is smaller than that of graphite (4.6-4.8 eV) [26], we use the value for graphite to enable direct comparison to previous reports in the literature, which typically assume a work function of $5 \mathrm{eV}$. This field enhancement factor is within the range typically reported for CVD-grown multi-walled carbon nanotube field emission sources $(\beta=400-1200)$ [12].

The excellent fit of experimental data to Fowler-Nordheim behavior indicates that space charge effects (which follow a $\mathrm{V}^{3 / 2}$ dependence) are unimportant for the CNT e-gun. This corroborates previous assertions in the literature [7,9]. 


\section{CONCLUSIONS}

Electron beams have been produced from two types of electron gun in the development of a miniaturized reflectron time-of-flight mass spectrometer. A conventional thermionic electron gun has been characterized electrically and using a commercially available beam visualization system. Experimental results have been compared to SIMION calculations.

A carbon nanotube-based cold cathode electron gun has been characterized electrically, and quantitative field emission data are well described by Fowler-Nordheim tunneling, as expected. In future work, we intend to characterize the shape of the electron beam produced by the CNT e-gun with modulation of the lens voltages, and the resultant observations will be correlated with SIMION predictions.

This research was supported by the Goddard Space Flight Center Internal Research and Development (IRAD) Program and the NASA Astrobiology Program. S.G. would like to thank Lance Delzeit for numerous helpful discussions.

\section{REFERENCES}

1. H H. B. Niemann, S. K. Atreya, S. J. Bauer, K. Biemann, B. Block, G. R. Carignan, T. M. Donahue, R. L. Frost, D. Gautier, J. A. Haberman, D. Harpold, D. M. Hunten, G. Isreal, J. I. Lunine, K. Mauersberger, T. C. Owen, F. Raulin, J. E. Richards, S.H. Way, Space Science Reviews 104, 570 (2002).

2. C. A. Spindt, I. Brodie, L. Humphrey, E. R. Westerberg, J. Appl. Phys. 47, 5428 (1976).

3. W. P. Dyke and W. W. Dolan, Adv. Electron. Electron. Phys. 8, 89 (1956).

4. A. G. Rinzler, J. H. Hafner, P. Nikolaev, L. Lou, S. G. Kim, D. Tomanek, P. Nordlander, D. T. Colbert, and R. E. Smalley, Science 269, 1550 (199.5).

5. Yu. V. Gulyaev, L. A. Chernozatonskii, Ja. Kosakovskaja, N. I. Sinitsyn, G. V. Torgashov, and Yu. F. Zakharchenko, J. Vac. Sci. Technol. B 13, 435 (1995).

6. W. A. de Heer, A. Chatelain, D. Ugarte, Science 270, 1179 (1995).

7. P. G. Collins and A. Zettl, Appl. Phys. Lett. 69, 1969 (1996).

8. Q. H. Wang, T. D. Corrigan, J. Y. Dai, R. P. H. Chang, and A. R. Krauss, Appl. Phys. Lett. 70, 3308 (1997).

9. J.-M. Bonard, J.-P. Salvetat, T. Stockli, W. A. de Heer, Laszlo Forro, and A. Chatelain, Appl. Phys. Lett. 73, 918 (1998).

10. W. Zhü, C. Bower, O. Zhou, G. Kochanski, and S. Jin, Appl. Phys. Lett. 75, 873 (1999).

11. S. Fan, M. G. Chapline, N. R. Franklin, T. W. Tombler, A. M. Cassell, and H. Dai, Science 283, 512 (1999).

12. X. Xu and G. R. Brandes, Appl. Phys. Lett. 74, 2549 (1999).

13. H. Murakami, M. Hirakawa, C. Tanaka, and H. Yamakawa, Appl. Phys. Lett. 76, 1776 (2000).

14. A. M. Rao, D. Jacques, R. C. Haddon, W. Zhu, C. Bower, and S. Jin, Appl. Phys. Lett. 76, 3813 (2000).

15. J. Cumings, A. Zettl, M. R. McCartney, and J. C. H. Spence, Phys. Rev. Lett. 88, 056804 (2002).

16. E. Minoux, O. Groening, K. B. K. Teo, S. H. Dalal, L. Gangloff, J.-P. Schnell, L. Hudanski, I. Y. Y. Bu, P. Vincent, P. Legagneux, G. A. J. Amaratunga, and W. I. Milne, Nano Lett. 5, 2135 (2005).

17. Y. Saito and S. Uemura, Carbon 38, 169 (2000).

18. C. Bower, W. Zhu, D. Shalom, D. Lopez, L. H. Chen, P. L. Gammel, and S. Jin, Appl. Phys. Lett. 80, 3820 (2002).

19. N. de Jonge and J.-M. Bonard, Phil. Trans. R. Soc. London A 362, 2239 (2004), and references therein.

20. W. I. Milne, K. B. K. Teo, M. Mann, I. Y. Y. Bu, G. A. J. Amaratunga, N. de Jonge, M. Allioux, J. T. Oostveen, P. Legagneux, E. Minoux, L. Gangloff, L. Hudanski, J.-P. Schnell, L. D. Dieumegard, F. Peauger, T. Wells, and M. El-Gomati, Phys. Stat. Sol. A 203, 1058 (2006).

21. V. H. Crespi, N. G. Chopra, M. L. Cohen, A. Zettl, and S. G. Louie, Phys. Rev. B 54, 5927 (1996).

22. SIMION 3D Ion and Electron Optics Simulator v8.0.2, Scientific Instrument Services, Inc., 1027 Old York Rd., Ringoes, NJ 08551, http://www.simion.com/.

23. L. Delzeit, C. V. Nguyen, B. Chen, R. Stevens, A. Cassell, J. Han, and M. Meyyappan, J. Phys. Chem. B 106, 5629 (2002).

24. R. H. Fowler and L. W. Nordheim, Proc. R. Soc. London, Ser. A 119, 173 (1928).

25. G. A. Amaratunga and S. R. P. Silva, Appl. Phys. Lett. 68, 2529 (1996).

26. R. Gao, Z. Pan, Z. L. Wang, Appl. Phys. Lett. 78, 1757 (2001). 